\title{
Comparative Genomics of Bacillus Subtilis MZK05 and Its Mutant Strain Revealed Genetic Factors Responsible for Enhanced Serine Protease Expression
}

\section{Md. Mozammel Hoq}

Fermentation and Enzyme and Biotechnology laboratory, Department of Microbiology, University of Dhaka, Dhaka-1000

\section{Md. Mahmud Hasan}

National Institute of Biotechnology, Ganakbari, Savar, Dhaka-1349

\section{Muhammad Manjurul Karim}

Fermentation and Enzyme and Biotechnology laboratory, Department of Microbiology, University of Dhaka, Dhaka-1000

\section{Md. Arafat Al Mamun}

Pilot Plant Research Laboratory, Centre for Advanced Research in Sciences, University of Dhaka, Dhaka1000

\section{Shakila Nargis Khan ( $\square$ shakila@du.ac.bd)}

Fermentation and Enzyme and Biotechnology laboratory, Department of Microbiology, University of Dhaka, Dhaka-1000

\section{Research Article}

Keywords: Complete genome sequence, Bacillus subtilis MZK05, Mutant M9, Serine protease, Signal peptidase

Posted Date: January 24th, 2022

DOI: https://doi.org/10.21203/rs.3.rs-1242999/v1

License: (c) (i) This work is licensed under a Creative Commons Attribution 4.0 International License. Read Full License 
1 Comparative genomics of Bacillus subtilis MZK05 and its mutant strain revealed genetic factors

2 responsible for enhanced serine protease expression

3

4 Md. Mozammel Hoq ${ }^{1}$, Md. Mahmud $\operatorname{Hasan}^{1,2}$, Muhammad Manjurul Karim¹, Md. Arafat Al Mamun ${ }^{1,3}$ 5 and Shakila Nargis Khan ${ }^{1 *}$

6

$7 \quad{ }^{1}$ Fermentation and Enzyme and Biotechnology laboratory, Department of Microbiology, University of 8 Dhaka, Dhaka-1000, Bangladesh

$9 \quad{ }^{2}$ National Institute of Biotechnology, Ganakbari, Savar, Dhaka-1349, Bangladesh

$10{ }^{3}$ Pilot Plant Research Laboratory, Centre for Advanced Research in Sciences, University of Dhaka, 11 Dhaka-1000, Bangladesh

12

13 Address correspondence: Shakila Nargis Khan, shakila@du.ac.bd.

14

15 
17 Genome sequence study of an industrially-important strain, Bacillus subtilis M9, a mutant version of wild 18 strain Bacillus subtilis MZK05 was conducted to uncover genetic factors responsible for enhanced serine 19 proteases expression in addition to its other industrial enzymes, metabolites and bacteriocins producing 20 efficacy. The wild type and the mutant genome contained a size of 4,145,727 and 4,045,950 bp, with 214,352 and 4,383 genes; and 477 and 478 subsystems respectively. Genomic comparison with $31 B$. 22 subtilis sourced from different countries showed both wild and mutant shared same type of genome 23 structure with 20 others. Moreover, 6,000 kb pangenome showed that they share 3082, 1449, and 25757

24 core, unique and accessory genes respectively. A sum of 32,559 mutations were found with three major 25 genomic structural changes in the upstream and downstream of an extracellular alkaline serine protease, $26 \mathrm{AprX}$ and a periplasmic serine protease, $\mathrm{HtrC}$ in $\mathrm{M} 9$ genome when compared to the wild type. 27 Furthermore, 11 different serine protease genes and 4 different signal peptidases were found with several 28 mutational changes in M9. In addition, mutations found in core genome of BsM9 in phage-like element, 29 major capsid protein, and phage portal protein are the likely reasons of high-level serine protease activity.

31 Keywords: Complete genome sequence, Bacillus subtilis MZK05, Mutant M9, Serine protease, Signal 32 peptidase 
43 Bacillus subtilis is a rod-shaped, Gram-positive, spore forming, nonpathogenic soil bacterium that 44 secretes numerous enzymes to degrade a variety of substrates, enabling the bacterium to survive in a 45 continuously changing environment. Proteases from Bacillus spp are one of the most commercially 46 valuable enzymes ${ }^{1}$ having enormous applications in food, feed, leather processing, detergent, digestive 47 aid and therapeutic purposes ${ }^{2}$. The genes coding for eight extracellular serine proteases were identified in 48 B. subtilis: $a p r E^{3,4}, b p r^{5,6}, e p r^{7,8}, m p r^{9,10}, n p r B^{11}, n p r E^{12}, v p r^{13}$, and $w p r A^{14}$. While two of them were 49 considered as major protease-coding genes, viz. aprE (alkaline serine protease subtilisin) and $n p r E$ 50 (neutral metalloprotease) accounting for $95 \%$ of the total extracellular protease ${ }^{15}$, the rest were minor 51 extracellular proteases. These enzymes help cells supplying amino acids for growth by degradation of 52 extracellular proteins ${ }^{16}$.

53 Earlier, we isolated eight Bacillus species from the effluents of tannery and poultry farms of 54 Bangladesh ${ }^{17}$, of which B. subtilis MZK05 (Bs MZK05) demonstrated potential extracellular enzymes, 55 identified as serine protease ${ }^{17}$. With a view to increasing the performance, the bacterium was subjected to 56 random mutagenesis using both chemical (ethyl methane sulfonate) and UV radiation ${ }^{18}$ that eventually 57 generated a mutant strain, B. subtilis M9 from a selection of potential strains, able to produce more 58 efficient extracellular protease than that of the wild type. This was demonstrated in unhairing and bating 59 of skin and hides, a performance comparable to that of the commercial enzymes, thereafter was applied 60 successfully in leather industries ${ }^{19},{ }^{20},{ }^{21}$. Therefore, we attempted here to compare the genome sequencing 61 of the two strains to pinpoint the probable cause of high-level expression of serine proteases in B. subtilis 62 M9 mutant, the number of proteases, and other industrially useful enzymes and metabolites, genes 63 responsible for extracellular protease secretion, genome variation in between the strains studied here with 64 that of strains from different countries of origin, and evolutionary relationships present with other 65 bacterial species of Bacillus subtilis group. 


\section{Results and Discussion}

\section{Genome sequence information}

70 Classification, general features, and genome sequencing information of B. subtilis MZK05 are tabulated

71 (Table 1) according to the MIxS recommendations. A total of 297,674 read pairs (average read length, 35-

72 301) and 372,189 read pairs (average read length, 35-301) were found with FastQC from B. subtilis

73 MZK05 and B. subtilis M9 respectively, and 37 contigs were obtained from each strain by SPAdes v.

$74 \quad 3.11 .1$ de novo genome assembly. N50 genome sequence of

75 B. subtilis MZK05 comprises a circular chromosome with a total size of 4,145,727 bp, and draft whole genome of $B$. subtilis M9 consists of $4,045,950 \mathrm{bp}$, and both the strains comprised of $43.5 \%$ of $\mathrm{G}+\mathrm{C}$ contents.

\begin{tabular}{|l|l|}
\hline Item & Description \\
\hline Organism & $\begin{array}{l}\text { Cellular organisms; Bacteria; Terrabacteria group; } \\
\text { Firmicutes; Bacilli; Bacillales; Bacillaceae; } \\
\text { Bacillus; Bacillus subtilis MZK05 }\end{array}$ \\
\hline Isolate type & Monoisolate \\
\hline Sample type & Chromosomal DNA \\
\hline Investigation type & Bacterial genome sequencing and annotation \\
\hline Project name & $\begin{array}{l}\text { Complete genome sequencing of Bacillus subtilis } \\
\text { MZK05 and Bacillus subtilis M9 }\end{array}$ \\
\hline $\begin{array}{l}\text { Geographic location of the } \\
\text { sampling site }\end{array}$ & $\begin{array}{l}\text { Hazaribagh, Dhaka City } \\
\text { Latitude 23.777176, Longitude 90.39942 }\end{array}$ \\
\hline $\begin{array}{l}\text { Geographic location (country } \\
\text { and/or sea, region) }\end{array}$ & Dhaka, Bangladesh \\
\hline Collection date & February, 2000 \\
\hline Environment (biome) & $\begin{array}{l}\text { Effluents discharge area of leather manufacturing } \\
\text { industries }\end{array}$ \\
\hline Environment (feature) & Effluent's liquids, mud and soil \\
\hline Environment (material) & $\begin{array}{l}\text { Hides \& skins processing waste liquid, muds, and } \\
\text { soils }\end{array}$ \\
\hline Environmental package & Liquids, muds and soils \\
\hline Sequencing method & Illumina Miseq \\
\hline Assembly method & SPAdes 3.11.1 \\
\hline Finishing quality & Finished (complete) \\
\hline &
\end{tabular}

79 Table 1. Classification, general features, and genome sequencing information of B. subtilis MZK05 and 
Gene function annotation, genomic features, variants and genome overview

83 The genetic features of B. subtilis MZK05 and B. subtilis M9 genome sequences, assembly, and

84 annotations are summarized in Table 2, and major subsystem features predicted from RAST are shown in

85 supplementary information (Figs. S1-S2, and Table S1). We found that both B. subtilis MZK05 and B.

86 subtilis M9 strains contain genes coding for industrially useful enzymes and metabolites, such as major

87 and minor serine proteases, signal peptidases, metalloproteases, alpha-amylase, phytase, xylanases,

88 pectate lyase, insulinase, bacteriocin-encoding genes (nisin, sublancin, lanthipeptide and subtilin) and

89 serine protease transcriptional regulators. Summary of the related results are shown in supplementary

90 information (Table S2). Circular graphical map of the genomes with COGs annotations are shown in Figs.

91 1a-b. Variant calling is an essential genome comparison method that provides insights into organismal

92 differences at nucleotide level. It comprises structural variants, single and multiple nucleotide

93 polymorphisms (SNPs, MNPs), insertions and deletions (indels). It coordinates genomes with

94 polymorphisms relative to a reference ${ }^{22}$ and estimates the impact of variants that occur in genes. Using

95 snippy 3.2-dev variant calling ${ }^{23}$, we have found 32,559 total variants, of them 3,814 Variant-COMPLEX,

96323 Variant-DEL, 304 Variant-INS, 5 Variant-MNP, and 28,113 Variant-SNP. Major genomic structural

97 differences between the wild and mutant Bs M9 genomes and variants are shown (Fig. 1).

\begin{tabular}{|l|l|l|}
\hline Features & Bacillus subtilis MZK05 & Bacillus subtilis M9 \\
\hline Base pairs (bp) & $4,145,727$ & $4,045,950$ \\
\hline GC content & $43.5 \%$ & $43.5 \%$ \\
\hline No of contigs & 37 & 37 \\
\hline N50 value of contigs & $1,045,489$ & 325,303 \\
\hline Longest contig length (bp) & $1,057,670$ & 612,811 \\
\hline Genes & 4,352 & 4,383 \\
\hline CDS (coding) & 4,140 & 4,130 \\
\hline Subsystems & 477 & 478 \\
\hline rRNAs & 12 & 9 \\
\hline tRNAs & 85 & 83 \\
\hline ncRNAs & 5 & 5 \\
\hline Pseudogenes (total) & 110 & 134 \\
\hline GenBank accession & CP032315 & RFFO00000000 \\
\hline SRA accession & SRR8199929 & SRR8200505 \\
\hline
\end{tabular}

98 Table 2. Summarized features of genome sequence, assembly and annotation of $B$. subtilis MZK05 and $B$.

99 subtilis $\mathrm{M} 9$ 


\section{Mutational changes in genome sequence}

101 The analysis of next generation genome sequence data yields major insights into the genetic features of

102 the organism ${ }^{24}, 25$. Here, we found that the mutations in the B. subtilis MZK05 strain produced nearly

103 about $0.1 \mathrm{Mb}$ reduced genome sequence than that of the mutant strain, B. subtilis M9. The related 104 mutational variants are shown in Fig. 1c and the major genetic changes are described in pangenome 105 section. From mauve genome alignment, it was clearly revealed that there were three major changes 106 occurred in very close region of two serine protease genes in B. subtilisM9. A frameshifted region was 107 identified in the downstream of a periplasmic serine protease $h t r C$ (Fig. 2a). Besides, deletions of about $10875 \mathrm{~kb}$ and $125 \mathrm{~kb}$ nucleotides were detected from upstream and downstream of an extracellular alkaline 109 serine protease $\operatorname{aprX}$ (Fig. 2b). These deletions occurred just before 17 genes and after 2 genes of aprX, 110 changes of which are thought to be linked with the increased production of serine protease in B. subtilis 111 M9. Similar to this finding, about $874 \mathrm{~kb}(20.7 \%)$ of the genomic sequence was deleted from B. subtilis 112 168, and the resulting mutant strain, B. subtilis strain MBG874 exhibited high level productivity of 113 extracellular cellulase and alkaline protease ${ }^{26}$. In another study, a deletion of $15 \%$ of the genome sequence $114(0.71 \mathrm{Mb})$ truncating all mobile DNA and cryptic virulence genes from an Escherichia coli strain yielded 115 some unexpected beneficial properties, yet ensuring normal cellular growth and protein expression ${ }^{27}$.

\section{Mutational changes in serine proteases}

118 There are 11 different serine protease genes present in both B. subtilis MZK05 and B. subtilis M9 (Figure $119 \mathrm{~S} 3)$. Amongst them, there are 2 major alkaline serine protease $(\operatorname{apr} X)$ genes with peptidase S8 family 120 domain, 1 minor serine protease (fibrinolytic protease Vpr) with inhibitor I9 and peptidase S8 subtilisin 121 Vpr-like family domain, and 1 trypsin-like extracellular serine metalloprotease (Mpr) (V8-like Glu122 specific endopeptidase). In addition, both wild and mutant strains had 1 intracellular serine protease (Isp), 1232 rhomboid family intramembrane serine protease (one of them is YqgP), 1 Bacillopeptidase F (Bpr) with 124 Peptidase S8 family domain with multiple catalytic active sites, and 3 membrane-bound and periplasmic 125 serine protease do-like genes $H t r A, H t r B$, and $H t r C$. Using MEGA-X protein alignment, we identified 
several mutations in different types of serine protease enzymes found in B. subtilis MZK05 and B. subtilis

127 M9 (Suppl. Mat. Table S3). Major structural changes in rhomboid family intramembrane serine protease 128 owing to mutation were detected in the serine protease of $B$. subtilis M9, particularly 3 changes in active 129 sites - S398N, E436K and N463Y; and 2 mutations at G359R and V376A that produced major and 130 noticeable 3D structural changes (Fig. 3a). There were four changes observed in extracellular serine 131 metalloprotease, one of which was Q88L that caused 3D structural change owing to loss of an alpha helix 132 (Fig. 3b). Two different sized AprX proteins were evident in both B. subtilis MZK05 and B. subtilis M9. 133 The larger AprX harbors 52 extra amino acids upstream of the coding sequence, the rest of the amino acid sequences were similar to both AprX. Two mutations were found in the small AprX in two different positions: N230S and T378A (Fig. 3c). The large AprX, on the other hand, had two substitutions: N282S and T430A (Fig. 3d). Remarkably, both of these large and small AprX had same type of two nonsynonymous changes: asparagine to serine at positions 282 and 230 (Fig. 3e); and threonine to alanine at positions 430 and 378 in large and small AprX respectively (Fig. 3f). Interestingly, the positions of the changes between the two varied sizes of AprX become alike if the stretch of upstream 52-amino-acid residues present in the large AprX is deducted. Further, 6 mutational changes were evident in Vpr, one of 141 these were T719S that brought a 3D structural change in the enzyme (Fig. 3g). Besides, 1 mutational 142 change was observed in Isp (Fig. 3h), and each in $\mathrm{HtrA}, \mathrm{HtrB}$, and $\mathrm{HtrC}$, however, no significant structural change was resulted due to these mutations. In addition, 7 mutational changes were found in 144 immune inhibitor A peptidase M6 region of bacillopeptidase F (Suppl. Mat. Table S3). Overall, the 145 deletion of upstream and downstream of an $\operatorname{aprX}$ gene (Fig. 2b), frameshift mutation in the downstream 146 of a periplasmic serine protease $h t r C$ (Fig. 2a), and multiple mutations in different types of serine 147 proteases genes, specially $a p r X, v p r, m p r$ and $y q g P$ in the B. subtilis $\mathrm{M} 9$ genome could be the likely 148 reasons for high-level enzymatic activity of alkaline serine protease in B. subtilis M9. On a relevant note, 149 as mutation in YqgP (GluP) region of rhomboid proteases altered glucose uptake and cell division in $B$. 150 subtilis $^{28,29}$, the presence of 15 mutations altering major structural changes in rhomboid protease YqgP of 
B. subtilis M9 could also be an important factor for high-level cell division and consequent serine

152 protease expression in B. subtilis M9.

\section{Observation in transcriptional regulators}

There were 6 different types of serine protease regulatory genes in both B. subtilis MZK05 and B. subtilis M9 identified as $\operatorname{deg} Q, \operatorname{cod} Y, \operatorname{lex} A, a b r B, y l b F$, and $\operatorname{marR}$ which are responsible for the expression of aprX, vpr, isp, mpr, yqgP, bpr, htrA, htrB, and htrC genes. No changes at the nucleotide level were observed in these regulatory genes.

\section{Mutational changes in signal peptidases}

We found that both B. subtilis MZK05 and B. subtilis M9 had 6 different serine types of signal peptidases (SPases) protein coding genes, and among them $\operatorname{sipI,~} \operatorname{sip} T$, $\operatorname{sip} V$ and 2 different $\operatorname{sipS}$ were from type I signal peptidase (SPase I) and a lipoprotein signal peptidase (lspA) from type II signal peptidase (SPase active catalytic sites: 100 (alanine), 101 (glutamine), and 102 (proline) resulting in alteration of the local

168 (amino acids 95 to 127) structure of SipV protein (Figs. 4a-b). The SipS, a signal peptidase of the S26 169 family, encountered 2 changes: L15T and S141T. While the later didn't have any impact, the former 170 affected the upstream protein structure until amino acid position 35 (Figs. 4c-d). SiPI produced 2 171 mutations: both of them influenced alteration of protein's 3D structure. These are T11S affecting amino 172 acid position 32 to 11 (Figs. 4e-f) and I179M affecting downstream terminal structure of the enzyme (Fig. 173 4e). Further, mutation at T64A of a second S26 family signal peptidase (Fig. 4g) altered most of the 3D 174 structure of the enzyme. This change affected catalytic sites: S47 and H87 (Fig. 4h). Overall, multiple 175 mutations in different serine type signal peptidases are thought to produce a high-level enzyme activity of 176 serine proteases in B. subtilis M9. 
178 From AntiSMASH $3.0^{30}$ and BAGEL4 ${ }^{31}$, four different classes of bacteriocin (nisin, sublancin, 179 lanthipeptide and subtilosin) encoding genes were found both in B. subtilis MZK05 and Bs M9. The 180 structure of bacteriocin genes with operon in MZK05 genome is illustrated in supplementary information, 181 Figure S4.

\section{Comparative genomics and phylogeny}

Comparative genomics of 31 complete genome sequences of $B$. subtilis strains from different countries, deposited in NCBI GenBank was addressed using Progressive Mauve genome alignment. Seven different types of structural and architectural patterns (Suppl. Mat. Fig. S5) were revealed; of them, 22 bacterial strains hailed from South Korea, Denmark, Germany, Greece, Belgium, China, India and USA including the present B. subtilis MZK05 and B. subtilis M9 strains grouped together to share common type of genome structure. Further analyses of these 31 B. subtilis strains by the BRIG-BLAST method presented a snapshot of the genetic diversity and close relatedness. The different color gradients of rings 4-34 (Suppl. Mat. Fig. S6), indicated a BLAST match of a percent identity in the key, located in the right side of the circular comparative genome map. From BRIG image, it was clearly evidenced that B. subtilis MZK05 had three major specific regions: 16.5 Mbp to 17.5 Mbp, 17.8 Mbp to 19.2 Mbp, and 24.2 to 25.5; and twenty-five small, distinctive segments scattered throughout B. subtilis MZK05 genome as compared to other strains. The symmetric identity of B. subtilis MZK05 with 30 other B. subtilis strains ranged from $70 \%$ to $90 \%$, and the greatest similarities were observed with strains reported from Germany

196 (ring 4, strain 168, CP019662), Belgium (ring 6, strain 2014-3557, CP045672), China (ring 8, strain 7PJ-

197 16, CP023409), India (ring 10, strain FB6-3, CP032089), South Korea (ring 13, strain HJ0-6, CP016894),

198 Japan (ring 16, strain NBRC 13719, AP019714), USA (ring 18, str. SMY, CP050532), South Korea (ring 199 23, strain SRCM102756, CP028218), China (ring 24, strain H1, CP026662), and South Korea (ring 28, strain SSJ-1, CP032860). Phylogenetic tree analysis revealed that bacterial strain B. subtilis MZK05 was grouped with B. subtilis strains (Suppl. Mat. Fig. S7) hence, the strain was identified as B. subtilis. The 
16S rRNA gene sequence of strain B. subtilis MZK05 is closely related to strains reported from South Korea, Denmark, Germany, Greece, Belgium, Egypt, Poland, China, Japan, South Africa, India and USA, generating $100 \%$ sequence identity. Besides, this strain is also very close to $B$. velezensis and $B$. amyloliquefaciens than B. licheniformis and B. paralicheniformis of Bacillus subtilis group bacteria.

\section{Pan-genome and COGs protein function analysis}

208 The NCBI-retrieved $30 \mathrm{~B}$. subtilis strains and the strains of interest of this study produced a 6,000 kb long pangenome (Fig. 6a), where each of the strains shared 3,082 core genes. Besides, 1,449 unique genes and 25,757 accessory genes were distributed in scattered manner among the strains. In pangenome, the position of the new genes in the different genomes are shown between 4,000 kb to 6,000 kb (just before the start position 0). The pan-versus-core gene plot clearly indicates that the pangenome window is still open but may be closed soon in the case of B. subtilis (Fig. 5a) and with the addition of each new genome to the $B$. subtilis multipart, a change in the pan-genome shape can result. Distribution of new genes within the pangenome of the B. subtilis genome are shown (Fig. 5b). Representative protein sequences of each strain were identified and their COGs and KEGG identities were determined. The details of core, accessory and unique genes present in each of the 30 genomes are shown (Suppl. Mat. Table S4). From the resulted table, it was found that 45 accessory genes were deleted or broken in B. subtilis M9 from $B$. subtilis MZK05. While the number of exclusively absent gene was 3 for B. subtilis MZK 05, the mutant lost additional 15 more genes making the figure 18 for B. subtilis M9. Besides, B. subtilis M9 had 43 extra unique genes that $B$. subtilis MZK05 and these genes were originated due to mutational breakdown

222 of accessory genes. From B. subtilis MZK05 and B. subtilis M9 core genome blast result, it was found 223 that major mutational changes were occurred in phage-like element PBSX protein XkdG, XkdM, XkdQ, 224 phage major capsid protein, phage portal protein, lytic transglycosylase, sulfate adenylyltransferase, 225 baseplate $\mathrm{J} / \mathrm{gp} 47$ family protein, LysM peptidoglycan-binding domain-containing protein, ABC 226 transporter ATP-binding protein and numerous hypothetical proteins (Suppl. Mat. Table S5). Previous

227 studies ${ }^{26,27}$ found that reduction of mobile element and genomic DNA increased high level productivity of 
extracellular cellulase, alkaline protease and beneficial properties. Likewise, reduction of some accessory

229 genes and mutation in core genes may increase the production and activity of alkaline serine proteases in

230 this study. The KEGG and COGs distribution of the representative proteins present in the core, accessory,

231 and unique genome are shown in Fig. 5c and Fig. 5d respectively, and the COGs frequency heatmap of

232 the representative proteins present in all of 30 genomes are shown (Fig. 5e). Relative evolutionary

233 divergence of Neighbor Joining (NJ) core and pan phylogenomic tree of 30 strains, constructed on the

234 basis of core genome and pan gene alignments are shown in (Figs. 6b-c). It was clearly revealed that the

235 position of $B$. subtilis MZK05 and B. subtilis M9 in core phylogenomic tree and pan phylogenomic tree

236 were different. In core phylogenomic tree B. subtilis MZK05 and B. subtilis M9 belonged to same node

237 with strains of China and Poland, whereas in pan phylogenomic tree mutant M9 strain moved to a new

238 position of another node with two South Korean strains. From core and pan phylogenomic tree analysis of

239 wild and mutant genomes, it was clearly revealed that mutational changes affected the accessory genes of

240 B. subtilis M9.

241

242 Conclusions

243 The comparative genomics of wild type B. subtilis MZK05 and its mutant strain M9 revealed underlying 244 genetic factors that prompted enhanced activity of serine protease in the mutant. Further, the analyses 245 provided insight for some very useful genes, coding for enzymes and metabolites, such as, major and 246 minor serine proteases, signal peptidases, metalloproteases, alpha-amylase, phytase, xylanases, pectate 247 lyase, insulinase, bacteriocin-encoding genes (nisin, sublancin, lanthipeptide and subtilin) and serine 248 protease transcriptional regulators, thereby making the strains industrially important for potential 249 bioeconomy.

\section{Data availability}

252 The complete genome sequence of the B. subtilis MZK05 and the draft whole genome sequence of $B$. 253 subtilis M9 are available at GenBank under the accession CP032315 and RFFO00000000 respectively. 
254 The raw data (Illumina) are available in the SRA under the accession number SRR8199929 and 255 SRR8200505, respectively.

Methods

259 Bacterial strains and genomic DNA preparation

260 The bacterial cultures of B. subtilis MZK05, previously isolated and identified (Hoq et al., 2005) and its 261 mutant B. subtilis M9 developed ${ }^{18}$ were prepared for sequencing by growing the strains separately 262 overnight at $37^{\circ} \mathrm{C}$ in $100 \mathrm{ml} \mathrm{LB}$ broth. Genomic DNA from B. subtilis MZK05 and B. subtilis M9 strains 263 were extracted with Thermo Scientific GeneJET Genomic DNA Purification Kit according to 264 manufacturer's instructions. Genomic DNA was quantified using NanoDrop One spectrophotometer 265 (Thermo Fisher Scientific, Waltham, MA, USA) and the integrity of the genomic DNA was analyzed by $2661 \%$ agarose gel electrophoresis. Then, a 300-bp paired-end library was constructed using the Nextera XT 267 DNA library preparation kit (Illumina) and bead-based normalization following the standard 268 manufacturer's protocol.

\section{Genome sequencing, and De novo assembly}

271 DNA of the strains (B. subtilis MZK05 and mutant B. subtilis M9) were sequenced with the Illumina 272 MiSeq technology (Illumina, San Diego, CA, USA) by MiSeq Reagent Kit v3 (600-cycle) at the Genome 273 Research Institute of North South University (NSU), Bangladesh. The quality of the raw reads was 274 checked with FastQC v. 0.11.7 $7^{32}$. Illumina adapter removal, read trimming and filtering were performed 275 using BaseSpace v3. Reads of both genomes were then assembled with SPAdes v. 3.11.1 ${ }^{33}$ in 'careful' 276 mode using the default $\mathrm{k}$ values of $21,33,55,77,99$ and 127 . Resulted contigs of both strains were 277 scaffolded using the move contigs command with default parameters of Mauve v. $2.4 .0^{34}, 35$, where the 278 genome of B. subtilis (CP021507) was used as reference. 


\section{Genome annotation, gene prediction and variant calling}

281 Automated functional annotation of scaffolded contigs of B. subtilis MZK05 and B. subtilis M9 was 282 performed by the RAST server ${ }^{36}$. Classic RAST v. 2.0 along with FIGfam (release 70) was used to 283 annotate both strains. Errors were fixed automatically. Gaps were backfilled and metabolic models were 284 created. Debug was not turned on and replication was disabled. Besides, final annotations were performed 285 using NCBI Prokaryotic Genome Annotation Pipeline (PGAP) ${ }^{37}$. Cluster of orthologous groups (COGs) protein function analysis and KEGG pathway were predicted by eggNOG-mapper v2 ${ }^{38}$ and WebMGA server $^{39}$. Circular graphical maps with COGs annotations were created by GView ${ }^{40}$. Wild and mutant bacterial genomic variants were detected by snippy 3.2- $\mathrm{dev}^{23}$ variant calling from NGS reads and overall genomic differences were observed by Progressive Mauve genome alignment ${ }^{34}$.

Identification of differences between serine protease genes in the genomes of the two strains. genes a genome-wide alignment with the Mauve alignment tool ${ }^{34}$ was performed where GenBank (.gb) file of the genome and mauve search tool were used to find out the position of serine protease genes in the aligned genomes. Structural changes of both nucleotide and amino acid sequences of serine proteases were also studied. Genetic level differences were studied by NCBI blastp and MEGA-X. 3D Pbd file of protein sequences were predicted by phyre $2^{41}$ webserver and structural and mutational changes were 298 studied by Pymol ${ }^{42}$.

\section{Identification of bacteriocin gene cluster}

301 The bacteriocin synthesizing gene clusters were revealed by AntiSMASH $3.0^{30}$ and BAGEL4 ${ }^{31}$.

\section{Comparative genome and phylogeny analysis}

304 Comparative genome and phylogenic analysis of closely related species of $B$. subtilis strain MZK05 and 305 M9 was performed by NCBI microbial genome BLAST. Best matched genome sequence of B. subtilis 
strains was analyzed and studied by two techniques, Mauve and Blast Ring Image Generator (BRIG).

Progressive Mauve command was used with default parameters to perform whole genome alignment with 29 best BLAST matched complete genome sequence of $B$. subtilis strains to reveal the genomic structural and architectural variations. Besides, best matched genome sequence of B. subtilis strains was also analyzed and studied by BRIG version 0.95 to determine overall genome comparison ${ }^{43}$. The circular comparative genomic map was constructed by BRIG with NCBI local blast-2.8.1+, with standard default

312 parameters. To determine the phylogenetic relationship, complete 16S rRNA gene sequences were 313 extracted from the genome sequences of 40 Bacillus strains $(24$ B. subtilis, 4 B. licheniformis, 4 B. paralicheniformis, 4 B. amyloliquifaciense, 4 B. velezensis) and were aligned using MUSCLE with default parameters. Phylogenetic tree was estimated by Maximum Likelihood Phylogeny statistical method with 1000 Bootstrap replications in MEGA-X with default parameters where substitution model and tree interference were developed by Tamura-Nei method and Nearest-Neighbor-Interchange (NNI) ML Heuristic Method respectively.

\section{Pangenome and COGs protein function analysis}

321 Pan- and core-genome analyses of 30 B. subtilis genomes were performed using GView ${ }^{40}$ and BPGA $322 \mathrm{v} 1.3^{44}$. GView was used to generate pangenome atlas as well as to study both pan and core genomes. 323 Besides, BPGA v1.3 was used with default clustering algorithm for orthologous gene identification and 324 clustering with a 50\% sequence identity cut-off. BPGA software uses USEARCH, CD-HIT, OrthoMCL 325 and MUSCLE software for the orthology analyses and powerlaw regression and exponential curve fit for 326 the pangenome, core genome developments, phylogeny, subset analysis, and KEGG \& COGs mapping.

327 Pangenome plot was extracted by plotting the total number of distinct gene families identified with each 328 subsequent addition of a genome against the number of genomes. Core-genome plot was extracted by 329 plotting the total number of shared genes with the addition of each genome against the number of 330 genomes. To avoid sampling biases, 30 iterations (random samples) were used for extracting these plots. 331 Representative sequences of both pan- and core-genomes were used for comprehensive functional 
analyses. Pan and core phylogeny were created from default parameter. COGs protein function of $30 \mathrm{~B}$. subtilis strains were predicted by WebMGA server ${ }^{39}$ and their comparative study were observed by $\mathrm{R}$ heatmap.

\section{References}

1. Rao, M.B., Tanksale, A.M., Ghatge, M.S. \& Deshpande, V.V. Molecular and biotechnological aspects of microbial proteases. Microbiol Mol Biol Rev. 62(3), 597-635 (1998).

2. Buescher, J.M. \& Margaritis, A. Microbial biosynthesis of polyglutamic acid biopolymer and applications in the biopharmaceutical, biomedical and food industries. Crit. Rev. Biotechnol. 27, 1-19 (2007).

3. Stahl, M.L. \& Ferrari, E. Replacement of the Bacillus subtilis subtilisin structural gene with an in vitro-derived deletion mutation. Journal of Bacteriology. 158(2), 411-418. ISSN 0021-9193 (1984).

4. Wong, S.L., Price, C.W., Goldfarb, D.S. \& DoI, R.H. The subtilisin E gene of Bacillus subtilis is transcribed from a sigma 37 promoter in vivo. Proceedings of the National Academy of Sciences. 81(4), 1184-1188. ISSN 0027-8424 (1984).

5. Sloma, A.L. et al. Bacillopeptidase F of Bacillus subtilis: purification of the protein and cloning of the gene. Journal of bacteriology.172(3), 1470-1477. ISSN 0021-9193 (1990).

6. Wu, X.C, Nathoo, S., Pang, A.S., Carne, T. \& Wong, S.L. Cloning, genetic organization, and characterization of a structural gene encoding bacillopeptidase F from Bacillus subtilis. Journal of Biological Chemistry. 265(12), 6845-50. ISSN 0021-9258 (1990).

7. Bruckner, R., Shoseyov, O. \& Doi, R.H. Multiple active forms of a novel serine protease from Bacillus subtilis. Molecular and General Genetics MGG. 221(3), 486-490. ISSN 0026-8925 (1990).

8. Sloma, A., Ally, A., Ally, D. \& Pero, J. Gene encoding a minor extracellular protease in Bacillus subtilis. Journal of Bacteriology. 170(12), 5557-5563. ISSN 0021-9193 (1988).

9. Rufo, G.A., Sullivan, B.J., Sloma, A.L. \& Pero, J.A. Isolation and characterization of a novel extracellular metalloprotease from Bacillus subtilis. Journal of Bacteriology. 172(2), 1019-1023, ISSN 0021-9193 (1990).

10. Sloma, A. et al. Gene encoding a novel extracellular metalloprotease in Bacillus subtilis. Journal of Bacteriology. 172(2), 1024-1029. ISSN 0021-9193 (1990). 
11. Tran, L.O., Wu, X.C. \& Wong, S.L. Cloning and expression of a novel protease gene encoding an extracellular neutral protease from Bacillus subtilis. Journal of bacteriology. 173(20), 6364-6372. ISSN 0021-9193 (1991).

12. Yang, M.Y., Ferrari, E.U. \& Henner, D.J. Cloning of the neutral protease gene of Bacillus subtilis and the use of the cloned gene to create an in vitro-derived deletion mutation. Journal of Bacteriology. 160(1), 15-21. ISSN 0021-9193 (1984).

13. Sloma, A.L. et al. Cloning and characterization of the gene for an additional extracellular serine protease of Bacillus subtilis. Journal of bacteriology.173(21), 6889-6895. ISSN 0021-9193 (1991).

14. Margot, P. \& Karamata, D. The wprA gene of Bacillus subtilis 168, expressed during exponential growth, encodes a cell-wall-associated protease. Microbiology. 142(12), 3437-3444, ISSN 13500872 (1996).

15. Kawamura, F. \& Doi, R.H. Construction of a Bacillus subtilis double mutant deficient in extracellular alkaline and neutral proteases. J Bacteriol. 160, 442-444 (1984).

16. Strauch, M.A. \& Hoch, J.A. Transition-state regulators: sentinels of Bacillus subtilis postexponential gene expression. Mol. Microbiol. 7, 337-342 (1993).

17. Hoq, M.M., Siddiquee, K.A.L., Kawasaki, H. \& Seki, T. Keratinolytic activity of some newly isolated Bacillus species. J. Biol. Sciences. 5(2), 193-200 (2005).

18. Salaheen, S., Mamun, M.A.A., Khan, S.N \& Hoq, M.M. Improvement of Bacillus licheniformis MZK05 by mutation for increased production of keratinase. Dhaka Univ. J. Biol. Sci. 24(1), 1723 (2015).

19. Mamun, M.A.A. et al. Development of an Alternative Enzyme-assisted Dehairing Method of Animal Skins using Proteases from Bacillus licheniformis MZK05M9. Bangladesh J. Microbiol. 2, 33-7 (2015).

20. Mamun, M.A.A., Khan, M.M., Akand, M.N., Khan, S.N. \& Hoq, M.M. Characterization of an alkaline protease with high quality bating potential in leather processing from Bacillus licheniformis MZK05M9 mutant. Intl. J. Biol. Res. 3(1):36-41 (2015).

21. Mamun, M.A.A., Mian, M.M., Saifuddin, M., Khan, S.N. \& Hoq, M.M. Optimization of fermenting medium by statistical method for production of alkaline protease by Bacillus licheniformis MZK05M9. J. Applied Biol. Biotechnol. 5(6), 24-28 (2017).

22. DePristo, M. A. et al. A framework for variation discovery and genotyping using next-generation DNA sequencing data. Nature Genet. 43, 491 (2011).

23. Seemann, T. Snippy: fast bacterial variant calling from NGS reads. Snippy: fast bacterial variant calling from NGS reads. Available online at: https://github.com/tseemann/snippy (2015). 
24. Kunst, F. The complete genome sequence of the gram-positive bacterium Bacillus subtilis. Nature. 390(6657), 249-256 (1997).

25. Hasan, M.M., Khan, S.N., Karim, M.M., Begum, A. \& Hoq MM. Complete genome and plasmid sequence of a novel Bacillus sp. BD59S, a parasporal protein synthesizing bacterium. 3 Biotech. 9(9), 318 (2019).

26. Morimoto, T. et al. Enhanced recombinant protein productivity by genome reduction in Bacillus subtilis. DNA Res. 15, 73-81 (2008).

27. Posfai, G. et al. Emergent properties of reduced-genome Escherichia coli. Science. 312, 10441046 (2006).

28. Mesak, L.R., Mesak, F.M. \& Dahl, M.K. Expression of a novel gene, gluP, is essential for normal Bacillus subtilis cell division and contributes to glucose export. BMC microbiology. 4(1), 13 (2004).

29. Rather, P. Role of rhomboid proteases in bacteria. Biochimica et Biophysica Acta (BBA)Biomembranes. 1828(12), 2849-2854 (2013).

30. Weber, T. et al. antiSMASH 3.0 - a comprehensive resource for the genome mining of biosynthetic gene clusters. Nucleic Acids Res. 43, 237-243 (2015).

31. Heel, A.J.V. et al. BAGEL4: a user-friendly web server to thoroughly mine RiPPs and bacteriocins. Nucleic Acids Res. 46, 278-281 (2018).

32. Andrews, S. FastQC: a quality control tool for high throughput sequence data. http://www.bioinformatics.babraham.ac.uk/projects/fastqc (2010).

33. Bankevich, A. et al. SPAdes: a new genome assembly algorithm and its applications to single-cell sequencing. J Comput Biol. 19, 455-477 (2012).

34. Darling, A.E., Mau, B. \& Perna, N.T. progressiveMauve: multiple genome alignment with gene gain, loss and rearrangement. PloS one. 5(6), e11147 (2010).

35. Darling, A.C., Mau, B., Blattner, F.R. \& Perna, N.T. Mauve: multiple alignment of conserved genomic sequence with rearrangements. Genome research. 14(7), 1394-1403 (2004).

36. Aziz, R.K. et al. The RAST Server: rapid annotations using subsystems technology. BMC genomics. 9(1), 75 (2008).

37. Tatusova, T. et al. NCBI prokaryotic genome annotation pipeline. Nucleic acids res. 44(14), 6614-24 (2016). 


\section{Competing interests}

454 The authors declare no competing interests. pipeline. Scientific reports. 6(1), 1-10.

\section{Acknowledgements} Government of the People's Republic of Bangladesh.

\section{Author contributions} authors read, and approved the manuscript.

38. Cantalapiedra, C.P. et al. eggNOG-mapper v2: Functional Annotation, Orthology Assignments, and Domain Prediction at the Metagenomic Scale. bioRxiv (2021).

39. Wu, S., Zhu, Z., Fu, L., Niu, B. \& Li, W. WebMGA: a customizable web server for fast metagenomic sequence analysis. BMC genomics. 12(1), 1-9 (2011).

40. Petkau, A., Stuart-Edwards, M., Stothard, P. \& Van Domselaar, G. Interactive microbial genome visualization with GView. Bioinformatics. 26(24), 3125-3126 (2010).

41. Kelley, L.A., Mezulis, S., Yates, C.M., Wass, M.N. \& Sternberg, M.J. The Phyre2 web portal for protein modeling, prediction and analysis. Nature protocols. 10(6), 845-858 (2015).

42. Lilkova, E. et al. The PyMOL Molecular Graphics System, Version 2.0 Schrodinger, LLC (2015).

43. Alikhan, N. F., Petty, N. K., Zakour, N. L. \& Beatson, S. A. BLAST Ring Image Generator (BRIG): simple prokaryote genome comparisons. BMC genomics, 12, 402 (2011).

44. Chaudhari, N. M., Gupta, V. K. \& Dutta, C. (2016). BPGA-an ultra-fast pan-genome analysis

This work was partially financed by the Ministry of Education (Ref: MoE/S-17/10 M-15/2007(Part1)/146) and Ministry of Science and Technology (Ref: MOSICT/S-9/B.Anu.Pr/86/2003-2004/386),

MMH isolated the organisms and supervised the experimental work. MMHasan analyzed the genome sequencing data, and drafted the manuscript. MMK did meticulous review of the manuscript. MAAM performed the supportive laboratory works for optimization of fermentation of alkaline serine proteases. SNK supervised the molecular biology part of the work, reviewed and finalized the manuscript. All 

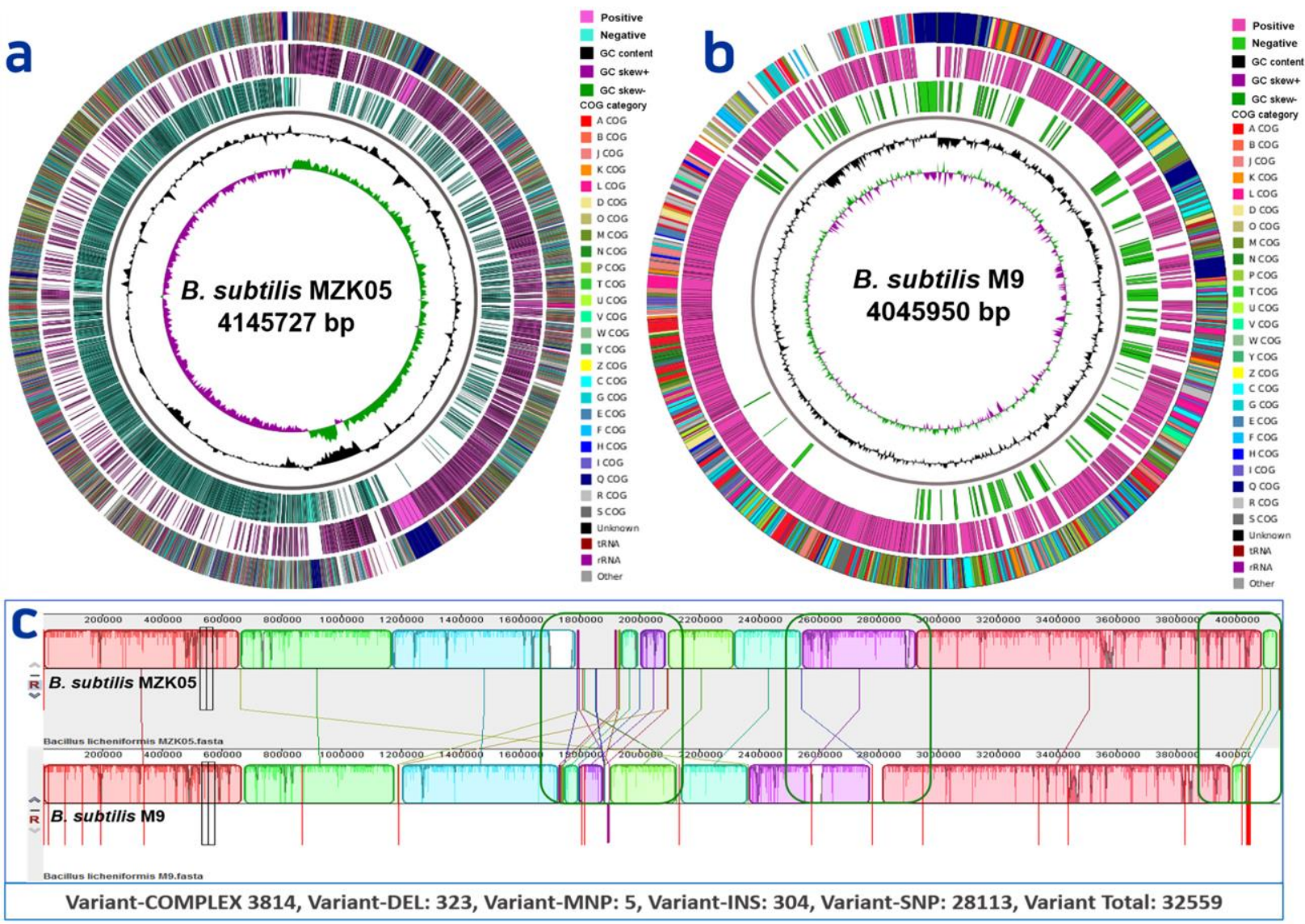

Figure 1. Circular graphical maps of B. subtilis MZK05 complete genome (a), and, B. subtilis M9 draft genome (b). Circles indicate, from inside to outwards: GC skew; GC content; total genes; rRNAs (green in chromosome); tRNAs (blue in chromosome); pseudogenes (sky blue); CDS on reverse strand (olive); CDS on forward strand (maroon); (c) Genome-wide alignment performed with Mauve progressive alignments compares the differences between B. subtilis MZK05 and B. subtilis M9 conserved genome regions. Each syntenical placement of the homologous region of the genome is represented as unique colored block, whilst divergent regions are seen as an empty block or line. Indentations within boxes highlight small mutations. Total number of variants after snippy 3.2-dev variant calling analyses are indicated beneath the alignment. 

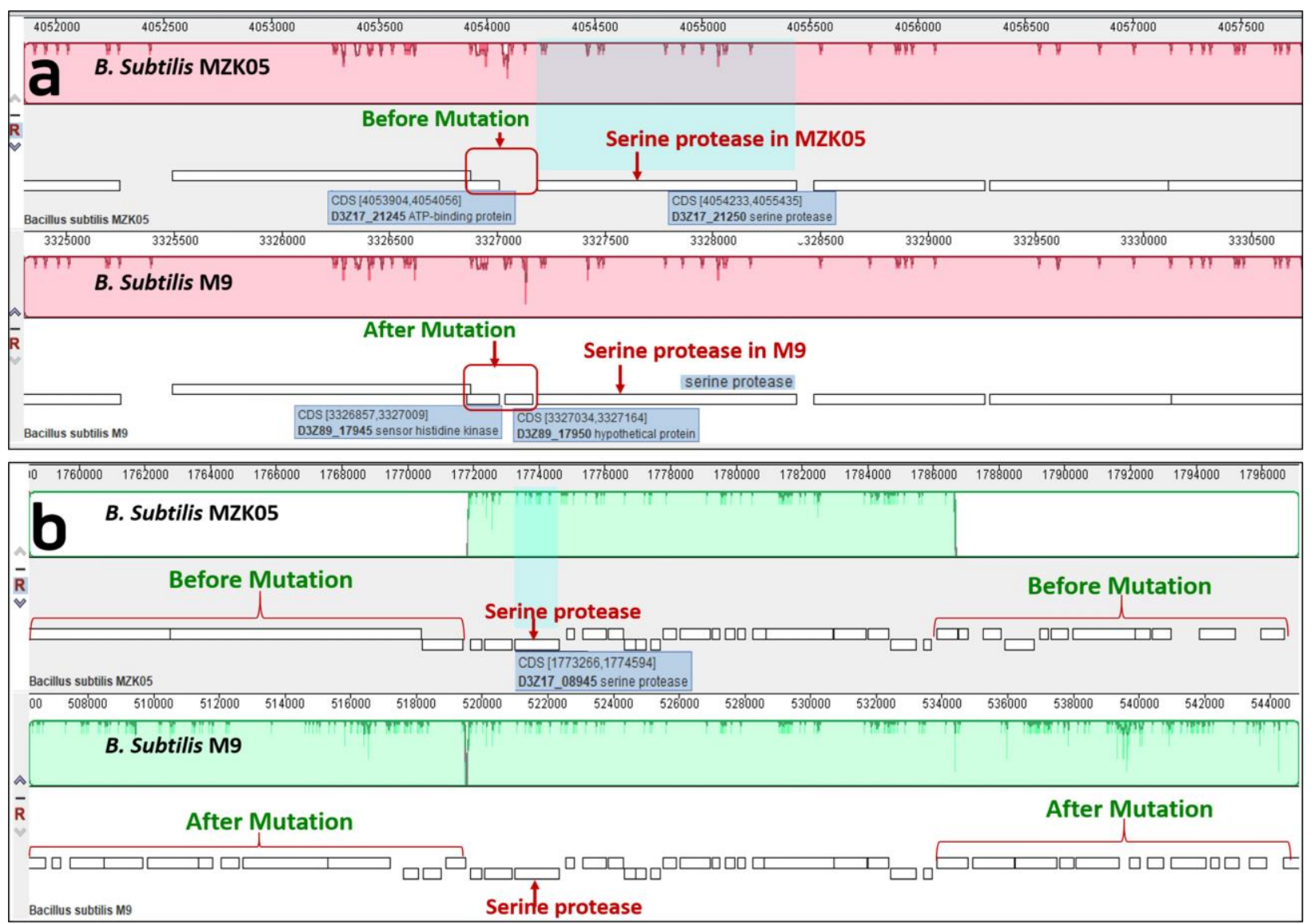

469 Figure 2. Multiple major changes observed in very close region of two serine protease genes. A single

470 mutated region is noticed just upstream of a serine protease gene (a), and in (b) Two large regions found 471 deleted just before and after another serine protease gene.

472

473

474

475

476

477 


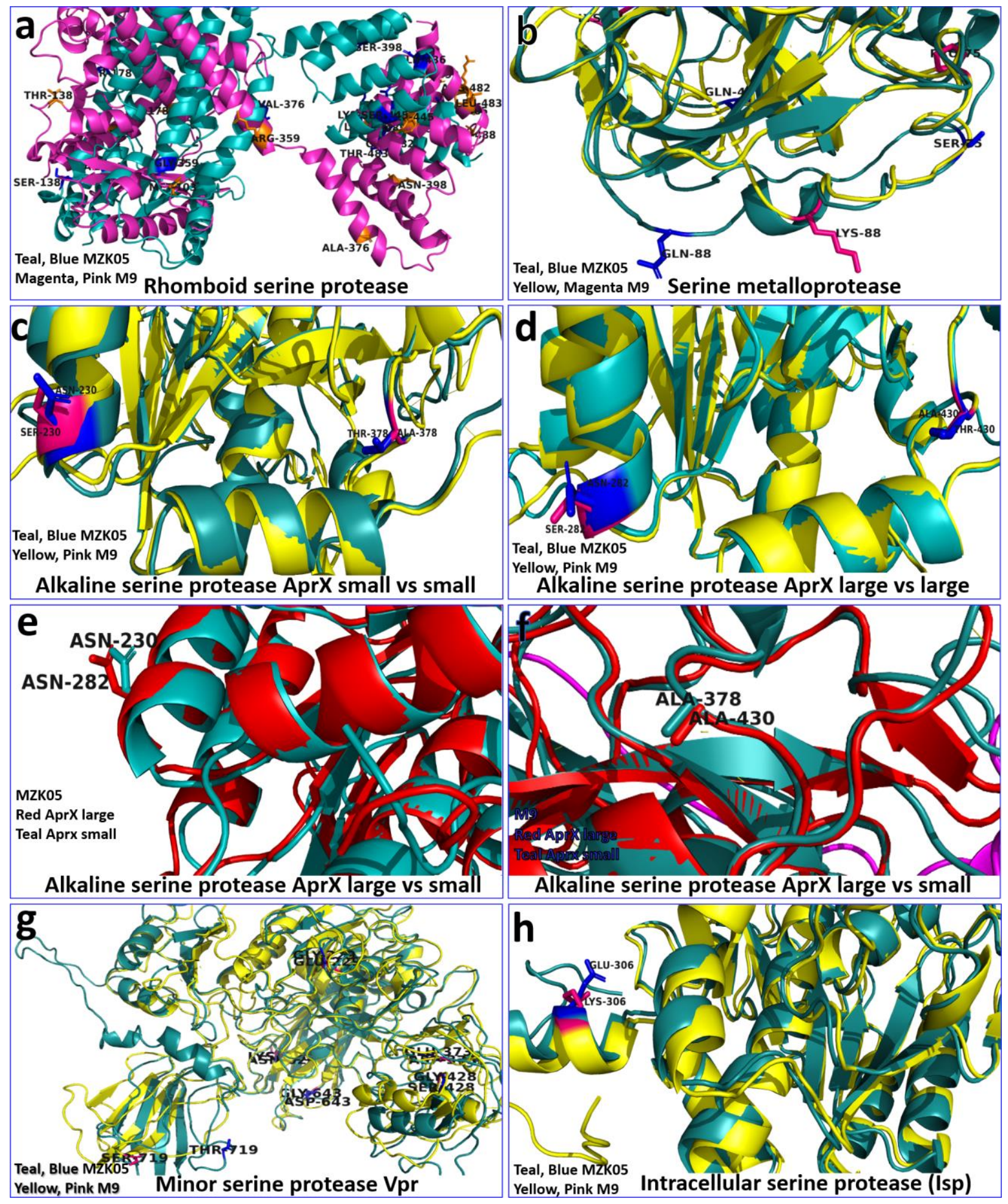

479 Figure 3. Depiction of amino acid mutations in serine proteases: (a) rhomboid serine protease, (b) 480 extracellular serine metalloprotease, (c-f) AprX, (g) Vpr, and (h) intracellular serine protease. 


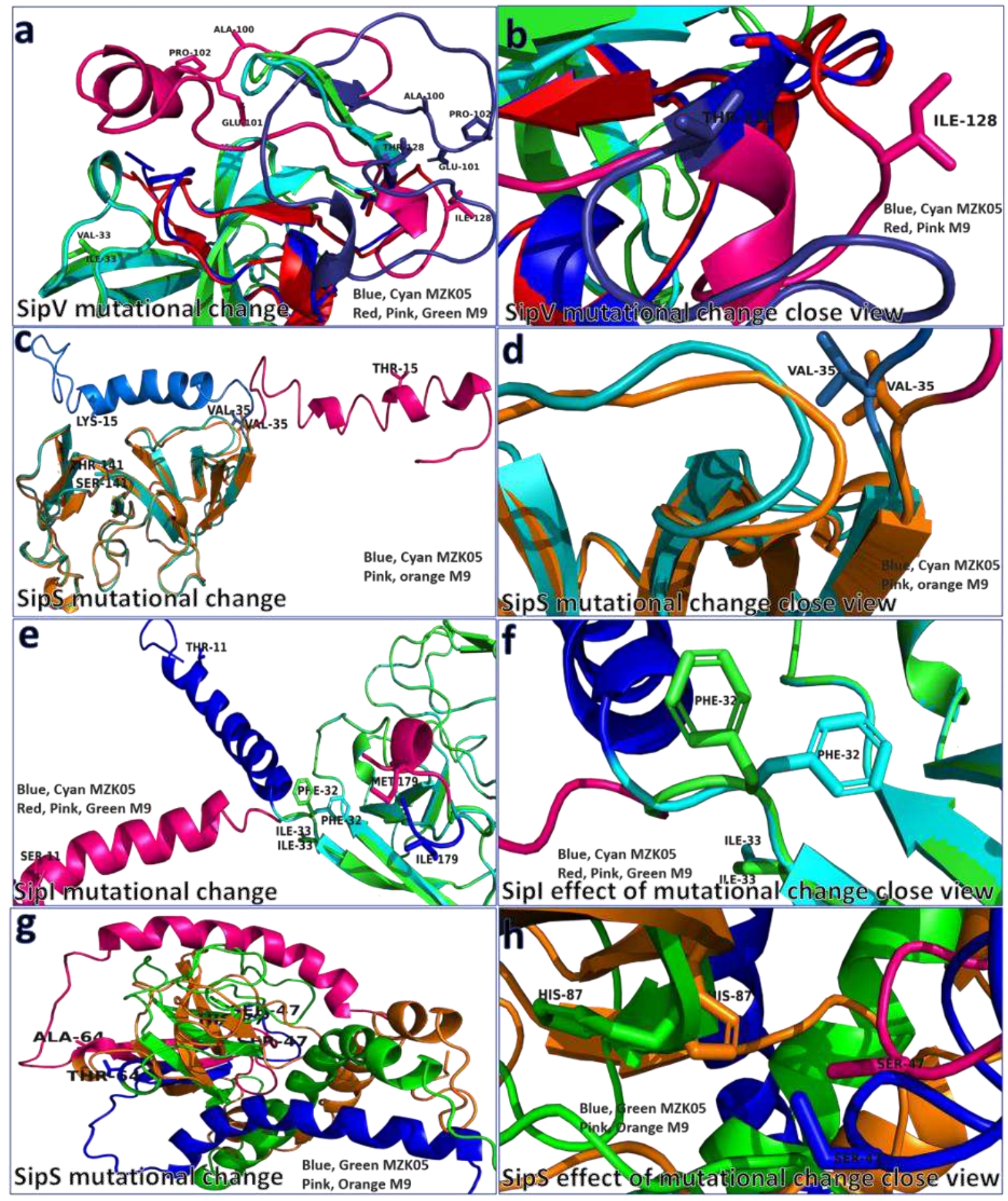

482 Figure 4. Mutational amino acid changes in signal peptidases (a-b) SipV, (c-d) SipS, (e-f) SipI, and (g-h) 483 SipS. 

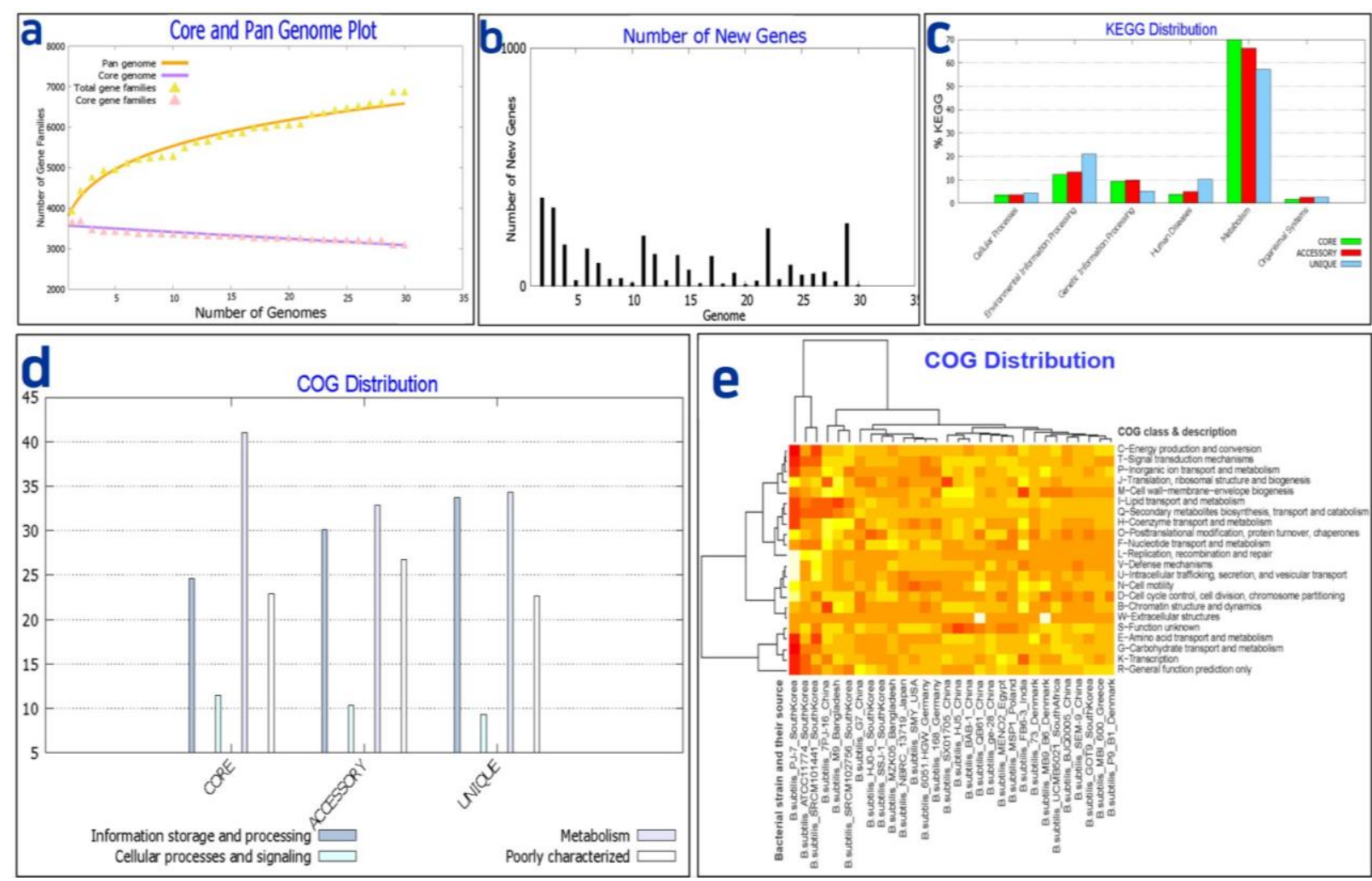

Figure 5. Pangenome plots of B. subtilis. (a) the pan and core genome box plot curves shows the evolution of the pangenome (blue) as an accumulated sum of gene families and the core genome (orange),

487 as described in the methods, (b) The columns give the number of new gene families introduced with the 488 addition of each genome into the consideration, (c) the KEGG and (d) COGs distribution of the 489 representative proteins in the core, accessory, and unique genome, and (e) The COGs frequency heatmap 490 of the representative proteins present in all 30 genomes, where, the vertical axis shows the abundance of 491 functional COGs category, and the strains are located on horizontal axis. 

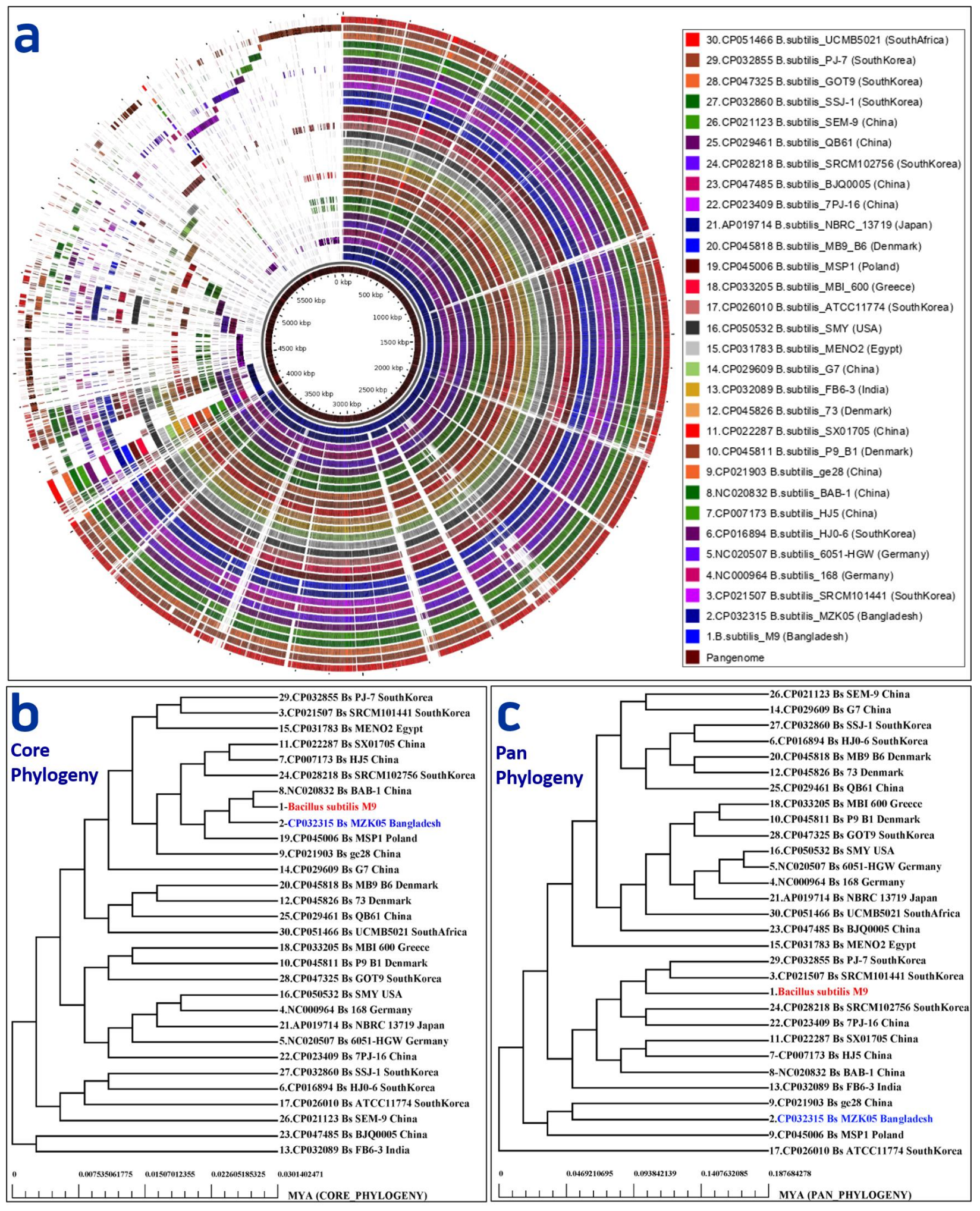

495 Figure 6. (a) Circular pangenome BLAST atlas of 30 B. subtilis genomes, where mutant strain B. subtilis

496 M9 was used as the reference strain. The Atlas shows diversities and new genomic properties as well as 
497 the genes of B. subtilis strains of different countries of origin. The inner most circle shows the pangenome 498 (Brown color). After pangenome, rings 1-2 represents B. subtilis M9 and B. subtilis MZK05 genome 499 respectively, rings 3-30: B. subtilis genome from different countries. The positions of different genomes 500 from inner to outward are mentioned in the legend of right-side box with names of the strain and countries 501 of origin. In pangenome, the position of the new genes in the different genomes are shown between 4000 $502 \mathrm{kbp}$ to $6000 \mathrm{kbp}$. Genomic regions unique to reference mutant strain and not appearing in other strains are 503 lightly colored and blank. (b) relative evolutionary divergence of B. subtilis, a Neighbor Joining (NJ) core 504 genome phylogenetic tree, and (c) a pan-genome phylogenetic tree, based on the pangenome of $30 B$. 505 subtilis strains. The bootstrap values are marked below position of each tree. Blue color indicates wild 506 strain B. subtilis MZK05, while red color indicates mutant strain B. subtilis M9. 


\section{Supplementary Files}

This is a list of supplementary files associated with this preprint. Click to download.

- SupplementaryinformationBacillussubtilisMZK05M9.pdf 